\title{
BMJ Open Healthcare resource use and costs of diabetic macular oedema for patients with antivascular endothelial growth factor versus a dexamethasone intravitreal implant in Korea: a population-based study
}

\author{
HyunJeong Cho, ${ }^{1}$ Kyung Seek Choi, ${ }^{2}$ Joo Yong Lee, ${ }^{3}$ Donghwan Lee, ${ }^{4}$ \\ Nam-Kyong Choi, ${ }^{5}$ YouKyung Lee, ${ }^{6}$ SeungJin Bae ${ }^{\oplus}$
}

To cite: Cho H, Choi KS, Lee JY, et al. Healthcare resource use and costs of diabetic macular oedema for patients with antivascular endothelial growth factor versus a dexamethasone intravitreal implant in Korea: a populationbased study. BMJ Open 2019;9:e030930. doi:10.1136/ bmjopen-2019-030930

- Prepublication history for this paper is available online. To view these files, please visit the journal online (http://dx.doi. org/10.1136/bmjopen-2019030930).

Received 09 April 2019 Revised 12 August 2019 Accepted 03 September 2019

Check for updates

(c) Author(s) (or their employer(s)) 2019. Re-use permitted under CC BY-NC. No commercial re-use. See rights and permissions. Published by BMJ.

For numbered affiliations see end of article.

Correspondence to

Dr SeungJin Bae;

sjbae@ewha.ac.kr

\section{ABSTRACT}

Objectives To estimate the costs and healthcare resources of patients with diabetic macular oedema (DME) who received intravitreal antivascular endothelial growth factor (anti-VEGF) agents or a dexamethasone intravitreal implant (DEX-implant) in Korea.

Design Retrospective cohort study.

Setting The Korean National Health Insurance claim data from 1 January 2015 to 30 June 2017 were retrieved from the Health Insurance Review and Assessment Service.

Participants Adult patients with DME who were diagnosed with diabetic retinopathy or DME and received ranibizumab, aflibercept or a DEX-implant in conjunction with intravitreal injection were included. Patients whose primary diagnoses were age-related macular degeneration or retinal vein occlusion were excluded.

Main outcome measures Healthcare resource utilisation and costs related to DME in the 12-month postindex period.

Results During the study period, 182 patients and 414 patients were identified in the anti-VEGF and DEXimplant groups, respectively, and there was no significant difference in the demographic characteristics between the two groups. The outpatient eye care-related medical costs were US\$3002.33 for the anti-VEGF group vs US\$2250.35 for the DEX-implant group $(p<0.0001)$. After adjusting the relevant covariates based on the generalised linear model, the estimated outpatient eye care-related medical costs were $33 \%$ higher in the anti-VEGF group than in the DEX-implant group ( $\mathrm{p}<0.0001,95 \% \mathrm{Cl} 22 \%$ to $45 \%$ ). The utilisation pattern of the two groups showed no significant difference except for the number of intravitreal injections, which was higher in the anti-VEGF group $(2.69 \pm 2.29)$ than in the DEX-implant group $(2.09 \pm 1.37, \mathrm{p}<0.001)$.

Conclusion The average annual eye-related medical cost of the DEX-implant group was significantly lower than that of the anti-VEGF group during the study period, which was mainly due to decreased utilisation of eye care-related injections. Further long-term studies are needed.
Strengths and limitations of this study

- Our study is the first attempt to investigate the healthcare resource utilisation of antivascular endothelial growth factor (anti-VEGF) versus the dexamethasone intravitreal implant (DEX)-implant among patients with diabetic macular oedema (DME) using population-based data in Asia.

- This study explored the relative healthcare utilisation of patients with DME and demonstrated that healthcare cost in the DEX-implant group was relatively cheaper than that in the anti-VEGF group.

- Various statistical approaches were applied to examine the robustness of the analysis.

- Due to the short study duration and off-label use of bevacizumab, further long-term studies using medical chart reviews should be conducted.

\section{INTRODUCTION}

Diabetic macular oedema (DME) is a common complication for patients with diabetes and the main cause of blindness in the non-elderly. ${ }^{1}$ Based on previous reports, $20 \%$ of patients with type 1 diabetes and $25 \%$ of patients with type II diabetes will suffer from DME; ${ }^{2}$ the 25 -year cumulative incidences of patients with DME and clinically significant DME for patients with diabetes are $29 \%$ and $17 \%$, respectively, and the risk of DME increases as the duration of diabetes increases. ${ }^{3}$ The number of adult patients with diabetes is expected to reach approximately 628.6 million in 2045 , which is a $50 \%$ increase from 424.9 million in $2017 .{ }^{4}$ According to the 2013-2016 Korea National Health and Nutritional Examination Survey (KNHANES), approximately 5 million Korean adults aged 30 years or older have diabetes, which 
comprises more than $1 / 3$ of the Korean population. ${ }^{5}$ DME could soon pose significant health concerns in Korea as well as around the world.

Blindness significantly reduces patients' quality of life and independence ${ }^{6}$ and is the third most serious health condition among the major complications of diabetes. ${ }^{7}$ Patients with DME reportedly have lower vision-related quality of life than do patients with diabetic retinopathy, patients with glaucoma and patients with cataracts. ${ }^{8}$

Additionally, patients with DME tend to have more comorbidities and consume more healthcare resources than do patients with diabetes without DME. ${ }^{9} 10$ The annual medical costs for patients with DME were approximately $30 \%$ higher than those for patients with diabetes without DME, and patients with DME were more than three times more likely to have ophthalmic visits after DME diagnosis (average 3.9 times) than were those without DME. ${ }^{11}$ Gonder and colleagues reached a similar conclusion and suggested that DME poses a significant economic and health burden in Canada. ${ }^{6}$

Treatment options for DME include laser photocoagulation, intravitreal agents such as antivascular endothelial growth factor (anti-VEGF) and steroids. ${ }^{12}$ Intravitreal injection is preferred over laser photocoagulation for the treatment of DME-associated visual impairment ${ }^{12} 13$ since patients with vision loss are less likely to experience significant improvements in best-corrected visual acuity after laser treatment, and improvement tended to slowly occur. $^{1415}$ Sustained-release corticosteroids have been developed to reduce the number of intraocular injections. The dexamethasone intravitreal implant (DEX-implant) is a sustained-release biodegradable implant indicated for the DME. ${ }^{16}$ Vascular endothelial growth factor (VEGF) is an important mediator of blood-retina barrier breakdown. ${ }^{17}$ Therefore, therapy that inhibits VEGF represents an effective therapeutic modality targeting the underlying pathogenesis of DME. ${ }^{14}$

Intravitreal injection of anti-VEGF agents is predominantly used for DME treatment, and two have been listed for DME by the Korean National Health Insurance (NHI): ranibizumab (Lucentis) since February 2015 and aflibercept (Eylea) since June 2015. ${ }^{18}$ Bevacizumab (Avastin), another anti-VEGF therapy, is used in Korea without regulatory approval (off-label use) and is not listed by the Korean NHI for DME. ${ }^{18}$ Sustained-release steroids made for intravitreal injection are another drug of choice for vision-impaired patients with DME, and the DEX-implant (Ozurdex), which falls into this category, has been listed in the Korean NHI since February 2016. ${ }^{18}$

Although both DEX-implant and anti-VEGF have shown similar efficacy in clinical trials, ${ }^{13}{ }^{19}$ they have different pros and cons. DEX-implant can be administered less frequently (every 4-6 months), ${ }^{16}$ whereas anti-VEGF may be administered every 4-6 weeks. ${ }^{15} 2021$ However, the DEX-implant is associated with an increased risk of cataracts or increased intraocular pressure. ${ }^{16}$ Thus, whether the DME-related healthcare resource utilisation of antiVEGF users is lower or higher than that of DEX-implant users is unclear. Given that DME causes a significant disease burden, it is important to evaluate the healthcare utilisation pattern of patients with DME between antiVEGF users and DEX-implant users. However, few studies have examined the healthcare resource use of these two groups using a population-based database.

We sought to compare the direct healthcare costs and the healthcare utilisation patterns of patients with DME using anti-VEGF (ranibizumab and aflibercept) or the DEX-implant by employing the Korean NHI database. Specifically, we compared the demographic characteristics and medical costs of the patients with DME using antiVEGF or the DEX-implant. Bevacizumab was not included since it was not listed by the Korean NHI for DME.

\section{MATERIALS AND METHODS \\ Data source}

We used the Korean NHI claim database, which covers approximately $97 \%$ of the Korean population of 48.4 million. The rest of the population is covered by the Medical Aid Program (MAP), a benefit programme for the underprivileged for which eligibility is determined based on patients' income level. ${ }^{22}$ The Health Insurance Review and Assessment Service (HIRA) provides access to the Korean NHI claim database with certain fees after the study protocol is evaluated. Access is allowed for a limited period for pre-specified individuals. ${ }^{23}$

The Korean NHI is reimbursed mainly based on the fee-for-service system, and the NHI claim data are administrative data collected by the NHI for the purpose of reimbursements. ${ }^{22}$ The NHI claim data contain demographic information, diagnosis codes based on the International Classification of Diseases-10th Revision (ICD-10), procedures codes, claim dates, cost of the drug/procedure/visit, and pharmacy claim records. Pharmacy claim records include the number of prescriptions filled during the study period, whereas the prescription information covers the date and duration of the prescription, the prescribed drugs' international non-proprietary names, dosage, route of administration and the prescribers' identification number. The patient identification numbers were removed, but a unique number was assigned for each patient so that patient-level data could be generated. ${ }^{22}$ We obtained patient-level data from 1 January 2015 to 30 June 2017, which covers the total Korean population with specific diagnoses, procedures, and medication records during that period. The study was approved by the Ewha Womans University Institutional Review Board (IRB File No. 150-1).

\section{Study population}

We conducted a retrospective cohort study from 1 January 2015 to 30 June 2017, to estimate the anti-VEGF agent or DEX-implant user's medical usage pattern and burden of disease. Patients aged 18 and older who were diagnosed with diabetic retinopathy (Korean classification of disease-7th revision (KCD-7) code H360) or retinal 
oedema (KCD-7 code H3580) based on their 5th diagnosis on the claim and received ranibizumab, aflibercept or DEX-implant in conjunction with intravitreal injection (Korean Current Procedural Terminology (CPT) code S5070.xx) in the index period (1 February 2016 to 30 June 2016) were included. Since we used the Korean NHI claim database and bevacizumab is not reimbursed for DME, we could not include bevacizumab. The index date or cohort entry date is the day that the patient with DME visited the ophthalmologic clinic during the index period and received the first study drug (ranibizumab, aflibercept or DEX-implant) with an intravitreal injection.

Patients with DME were classified as the anti-VEGF cohort and the DEX-implant cohort based on their medication utilisation pattern in the index period, and those patients who received the same medication in the postindex period and made at least three ophthalmic-outpatient visits during the follow-up period (1 year from the index date) were analysed for our analysis. Since patients with DME need to be monitored at least quarterly, we included patients who had at least three ophthalmic visits as the study populations. Medication claim records whose primary diagnoses were age-related macular degeneration (KCD-7 code H3530, H3531, and H3539) or retinal vein occlusion (KCD-7 code H348 and H349) were excluded for the analyses. We set the index period to reflect the Korean NHI listing date of the DEX-implant in Korea (1 February 2016).

Patients' baseline characteristics, such as age and gender, were measured at the index date. The Charlson comorbidity index (CCI), which measures a patient's comorbidity and is frequently used to adjust a patient's comorbidity, was measured in the preindex period, which is 1 year before the index date. ${ }^{24}$ The provider's specialty and the types of medical institutions visited by patients with DME were also measured at baseline. The study design is shown in figure 1 .

\section{Outcomes}

The primary outcome was the total annual medical cost per patient with DME that was reimbursed by the Korean NHI. Any healthcare utilisation that occurred for patients with DME during the 12 month postindex period was aggregated to estimate the total healthcare costs, and then this healthcare utilisation was categorised as outpatient/eye care-related utilisation based on the claim category (eg, outpatient) and provider's specialty (eg, ophthalmologist) on the claim. Namely, outpatient eye care-related medical costs were estimated by summing all of the claims that occurred for the patient with DME in an outpatient setting by the ophthalmologist during the postindex period. The monetary value was estimated based on Korean won (KRW) with US $\$ 1$ translating into approximately $1100 \mathrm{KRW}$ as of May 2018.

The secondary outcome was the number of visits and number of DME-related therapeutic and diagnostic procedures received per patient with DME during the postindex period. Optical coherence tomography (Korean CPT code EZ796), fluorescein angiography (FA) (Korean CPT code E6681 and E6682), fundus examination (Korean CPT code E6660), fundus photography (Korean CPT code E6670 and E6674), tonometry (Korean CPT code E6751 and E6752), slit-lamp biomicroscopy (Korean CPT code E6810) and intravitreal injection (Korean CPT code S5070.xx) that occurred during the postindex period were aggregated per patient with DME and compared between the two cohorts.

\section{Statistical analysis}

We used descriptive statistics to assess differences between patients who had anti-VEGF or the DEX-implant using $\chi^{2}$ tests and Fisher's exact tests. If the cell sizes were less than 5, then we used Fisher's exact test. Because the medical costs and visit days were skewed, we specified a $\log$ link with gamma distribution. ${ }^{25}$ We analysed the likelihood-ratio test for the generalised linear model (GLMs) for the variables related to costs and visit days. To estimate the unadjusted and adjusted effects of the two groups on the medical cost and visit days, we used GLMs. ${ }^{26}$ For the adjusted effects, we adjusted the baseline characteristics such as age, gender, CCI and health plan type. These distributions of medical costs and visit days are non-parametric; thus, we evaluated healthcare utilisation using the Wilcoxon rank sum test. However, there was also a previous study regarding cost analysis that suggested a t-test in the guideline for comparing the average cost of the two groups; ${ }^{27}$ thus, we presented both the t-test (for parametric) and Wilcoxon rank sum test (for non-parametric) for the costs and resource use to examine the robustness of the results. SAS software V.9.4 (SAS Institute, Cary, North Carolina, USA) was used for the analysis.

\section{Patient and public involvement}

Since we used the NHI claim database, individual patients were not involved in the study design. We could not disseminate the results of the research directly to study

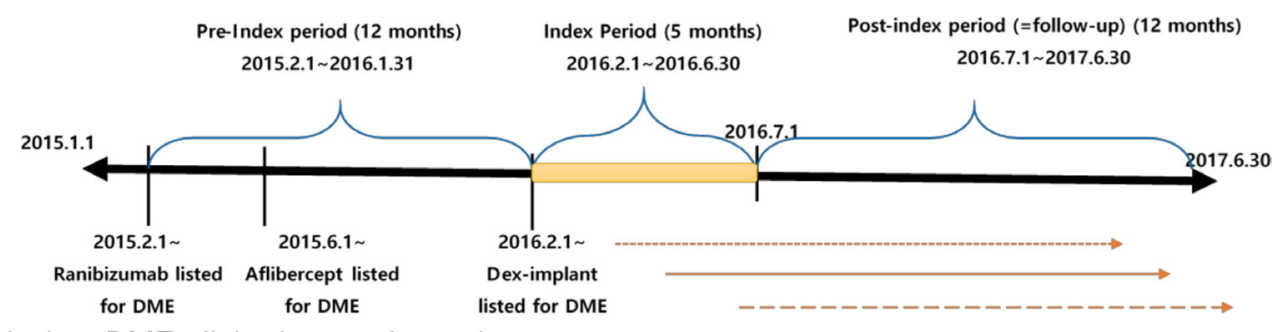

Figure 1 Study design. DME, diabetic macular oedema. 
700

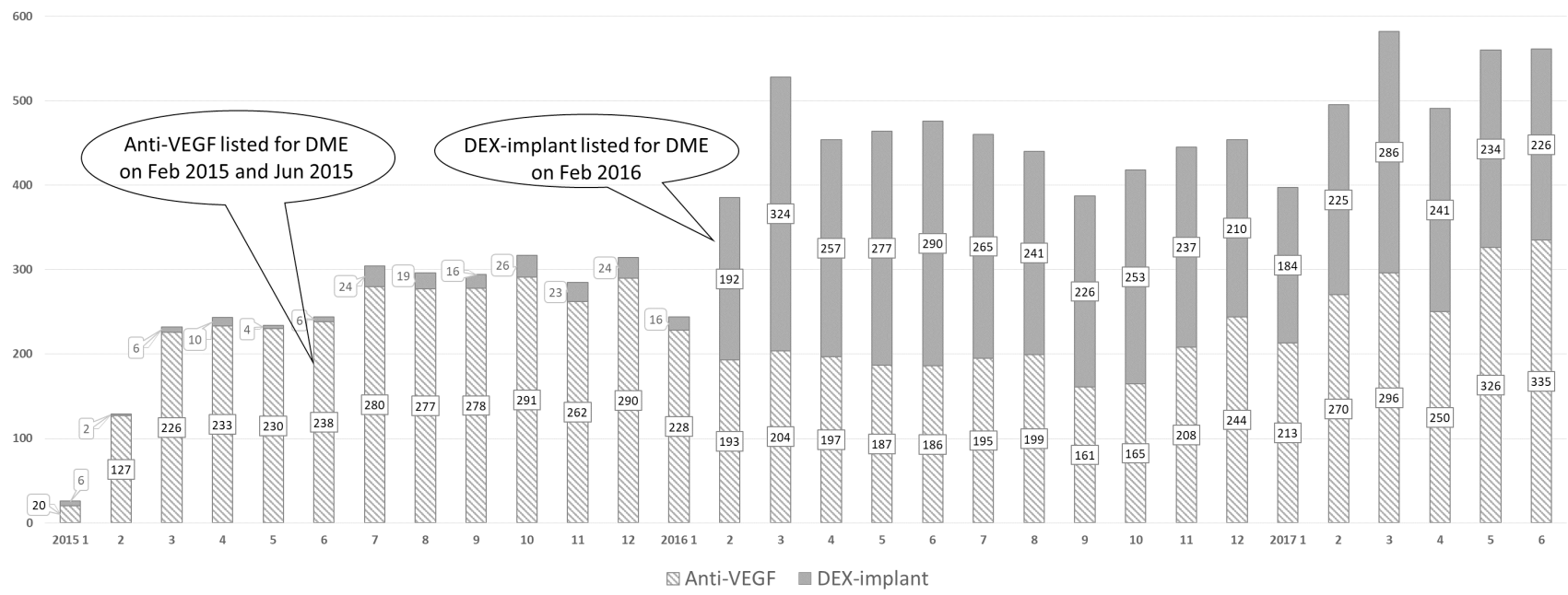

Figure 2 The number of patients with DME who received either anti-VEGF or a DEX-implant from 1.1.2015 to 30.6.2017. AntiVEGF includes ranibizumab and aflibercept. Anti-VEGF, antivascular endothelial growth factor; DEX-implant, dexamethasone intravitreal implant; DME, diabetic macular oedema.

participants since patients' identifiable information was blocked by the HIRA.

\section{RESULTS}

During the study period, the number of monthly patients with DME who were treated with anti-VEGF or the DEX-implant increased from 26 patients in January 2015 to 561 patients in June 2017. With the DEX-implant listed since February 2016, the number of patients with DME using either anti-VEGF or the DEX-implant soared (figure 2).

For our cohort analysis, a total of 1486 patients with DME were treated with anti-VEGF agents (ranibizumab or aflibercept) or the DEX-implant during the index period. Of those 1486 patients with DME, only 596 were included for the analysis; 182 patients were treated with anti-VEGF, and 414 patients were treated with the DEX-implant alone during the follow-up period (890 of these patients were not included in the analysis). Of those 890 excluded patients, 727 did not receive any study medication during the follow-up period, although they had received the study medication during the index period at least once. We could not analyse these patients because there were no data for the follow-up period. A total of 109 of these patients switched the medication they received in the index period to the other group's medication during the follow-up period. Those patients were excluded because their switching pattern varied greatly, and the clinical rationale behind switching could not be found based on the claim database. Total 54 of these patients had fewer than three ophthalmic-outpatient visits (data not shown).
The demographic characteristics of the study population are shown in table 1 . The average age for the antiVEGF cohort and DEX-implant cohort was 58.6 vs 59.6, respectively, with no significant difference observed $(\mathrm{p}=0.406)$. Gender distribution and CCI distribution also showed no significant difference between the two groups. Although the CCI scores, which were measured 12 months prior to the index date, showed no significant difference $(\mathrm{p}=0.083)$, a higher percentage of anti-VEGF users had a lower score. In other words, $18.1 \%$ of the antiVEGF and $10.6 \%$ of the DEX-implant fall into category 0 , which indicates that a higher portion of anti-VEGF patients had fewer comorbidities (table 1). The proportion of anti-VEGF group patients $(13.7 \%)$ insured under the MAP was significantly higher than that of DEX-implant group patients $(5.1 \%, \mathrm{p}=0.0003)$.

Table 2 shows the total medical cost, outpatient-medical cost, eye care-related medical cost, and outpatient-medical cost for ophthalmology for 12 months. Overall, the average total medical cost per person for the follow-up period was US\$6331.86 for the anti-VEGF group and US\$5708.54 for the DEX-implant group. The outpatient eye care-related medical cost was significantly different in the average medical cost (t-test, $\mathrm{p}<0.0001)$ and median medical cost (Wilcoxon rank sum test, $\mathrm{p}=0.0012$ ), with a mean value of US $\$ 3002.33$ for the anti-VEGF group and US\$2250.35 for the DEX-implant group (table 2). After adjustment for age, gender, CCI and health plan type, the outpatient-medical cost and outpatient eye care-related medical cost were $20 \%$ and $33 \%$ higher in the antiVEGF group than in the DEX-implant group, respectively $(\mathrm{p}<0.0001)$. 
Table 1 Demographic characteristics of patients with DME who received either anti-VEGF agents or a DEX-implant during the study period

\begin{tabular}{|c|c|c|c|}
\hline Variable & $\begin{array}{l}\text { Anti-VEGF } \\
(n=182)\end{array}$ & $\begin{array}{l}\text { DEX-implant } \\
(n=414)\end{array}$ & $P$ value \\
\hline $\begin{array}{l}\text { Age, mean (SD), } \\
\text { years }\end{array}$ & $58.6(12.6)$ & $59.6(10.8)$ & 0.406 \\
\hline Age, $n(\%)$, years & & & 0.409 \\
\hline $18-39$ & $10(5.5)$ & $22(5.3)$ & \\
\hline $40-49$ & $28(15.4)$ & 46 (11.1) & \\
\hline $50-59$ & $54(29.7)$ & $127(30.7)$ & \\
\hline $60-69$ & $54(29.7)$ & $149(36.0)$ & \\
\hline $70+$ & $36(19.8)$ & 70 (16.9) & \\
\hline Gender, n (\%) & & & 0.197 \\
\hline Male & $101(55.5)$ & $206(49.8)$ & \\
\hline Female & $81(44.5)$ & $208(50.2)$ & \\
\hline $\mathrm{CCl}, \mathrm{n}(\%)$ & & & 0.083 \\
\hline 0 & $33(18.1)$ & 44 (10.6) & \\
\hline 1 & $55(30.2)$ & $130(31.4)$ & \\
\hline 2 & $43(23.6)$ & $103(24.9)$ & \\
\hline 3 & $51(28.0)$ & $137(33.1)$ & \\
\hline $\begin{array}{l}\text { Health plan type, } n \\
(\%)^{*}\end{array}$ & & & 0.0003 \\
\hline $\mathrm{NHI}$ & 157 (86.3) & 393 (94.9) & \\
\hline MAP & $25(13.7)$ & $21(5.1)$ & \\
\hline
\end{tabular}

Age, gender and health plan type are analysed based on the index date. The $\mathrm{CCl}$ score is calculated for the 12 months from the start of the index date. $P$ value estimated based on the $\chi^{2}$ (categorical variables) and t-test (continuous variable). *Korea has the NHI system for the general population and the MAP for the low-income people. The NHI system is for the entire population residing within the territory of Korea except for beneficiaries of medical aid.

anti-VEGF, antivascular endothelial growth factor; CCl, Charlson Comorbidity Index; DEX-implant, dexamethasone intravitreal implant; DME, diabetic macular oedema; MAP, Medical Aid Program; NHI, National Health Insurance.

Table 3 shows the healthcare visit days for the 12-month follow-up period between the two groups. The number of visits, such as the total number of visits, outpatient visits, and outpatient eye care-related visits, was not significantly different between the two groups.

Table 4 illustrates no significant difference in utilisation pattern between the two groups, except intravitreal injection, where both parametric $(\mathrm{p}<0.001)$ and non-parametric $(p<0.0044)$ approaches achieved consistent results. Although significance was not achieved, the DEX-implant showed higher utilisation in tonometry based on the mean (12.08 vs 13.21) and median (11 vs 12).

\section{DISCUSSION}

We compared real-world healthcare utilisation of DME cohorts who were treated with anti-VEGF or the
DEX-implant and using data from the Korean NHI claim database. Although the number of patients in each cohort was rather limited, the average annual healthcare cost for patients with DME was approximately 6.9 million KRW (or US\$6331) for the anti-VEGF group and 6.2 million KRW (or US \$5708) for the DEX-implant group. Given that the annual cost per diabetes patient was 3.3 million KRW as of $2013,{ }^{28}$ the burden of DME is substantial.

However, there are few studies investigating the burden of patients with DME using population-based data, much less in Asia. In Canada, one prospective observational study on 145 patients investigated the DME cost by collecting the patients' medical charts and concluded that the average 6-month DME-related cost per patient was US $\$ 2092$, which translates into US $\$ 4184$ over 12 months. ${ }^{6}$ Given that our average annual eye care-related medical cost ranges from 3.4 million KRW (or US\$3161) for the anti-VEGF group and 3 million KRW (or US\$2751) for the DEX-implant group, our estimate is consistent with that of the Canadian study. Additionally, the Canadian study is based on a medical chart review, whereas our study is based on the administrative claim database; thus, our data could not incorporate non-reimbursed services and off-label use, such as bevacizumab. Bevacizumab is not indicated for DME and therefore is not listed by the Korean NHI; however, it is reportedly widely used due to its economic attractiveness. Thus, the two cohorts in our analyses might include patients with DME who were exposed to bevacizumab during the study period.

Further long-term studies are needed to investigate the relative utilisation of bevacizumab in two cohorts by examining medical charts. However, we assumed that the inclusion of bevacizumab would not significantly alter our findings since a previous study ${ }^{29}$ based on a medical chart review showed that the utilisation pattern of bevacizumab would be similar to that of other anti-VEGF agents. Additionally, the rate of combination therapy (ie, bevacizumab +other anti-VEGF or bevacizumab +DEX-implant) would be low.

Additionally, our study was population-based; thus, our research does not suffer from selection bias, and the patient number is four times larger than that of the Canadian study. ${ }^{6}$ Few studies have examined the healthcare resource utilisation of anti-VEGF versus DEX-implant groups using population-based data. One US study compared bevacizumab versus the DEX-implant in 50 eyes and suggested that the number of injections in the DEX-implant group was significantly lower than that in the bevacizumab group. ${ }^{30}$ Although our study medication does not include bevacizumab since it is not listed in the Korean NHI, one could argue that our result is consistent with the results of Shah and colleagues since bevacizumab is in a class of anti-VEGF agents. Given that the DEX-implant reportedly has a longer duration, ${ }^{31}$ our result is not surprising. The difference between two cohorts in the number of injections is not as dramatic as that in previous studies, which might be because aflibercept was included 
Table 2 Annual healthcare cost for patients with DME who received either anti-VEGF agents or a DEX-implant

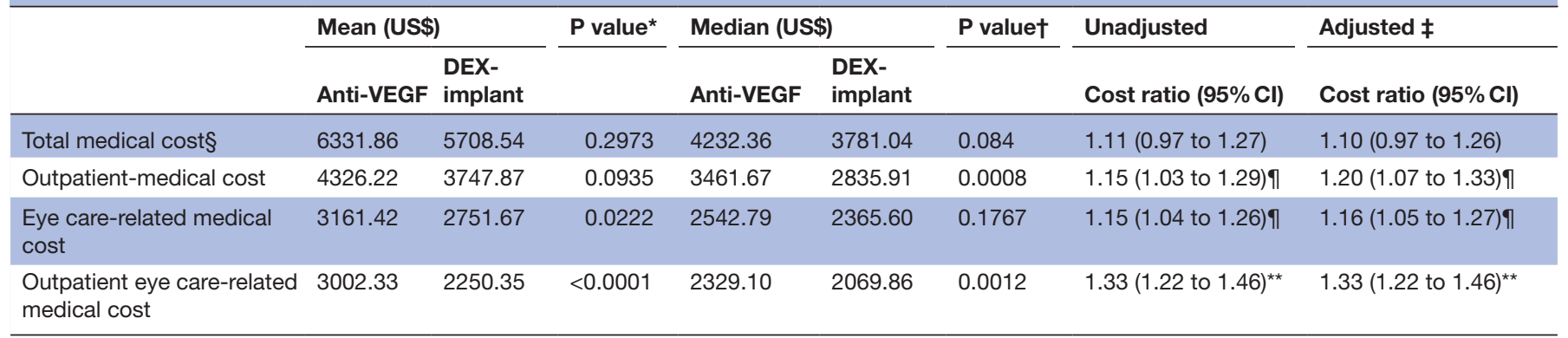

Anti-VEGF includes ranibizumab and aflibercept only. The total cost of medical care is the aggregated cost of all medical care used by the patient during a 1-year follow-up period. The cost was adjusted as of May 2018 in US dollars (US\$1 equals 1100 Korean won).

*Estimated $p$ values based on the t-test.

tEstimated $p$ values based on the Wilcoxon test.

$\ddagger$ Adjusted for baseline characteristics such as age, gender, $\mathrm{CCl}$ and health plan type.

$\S$ The cost of the DEX-implant group being referenced (1).

IP $<0.001$.

${ }^{* \star} \mathrm{P}<0.05$.

anti-VEGF, antivascular endothelial growth factor; CCI, Charlson Comorbidity Index; DEX-implant, dexamethasone intravitreal implant; DME, diabetic macular oedema.

in our analysis, which reportedly has a longer duration than that of other anti-VEGF agents. ${ }^{32}$

Our results suggested that the proportion of anti-VEGF group patients insured under the MAP was significantly higher than that of the DEX-implant group. Since the DEX-implant is more likely to be used by tertiary or teaching hospitals, whereas anti-VEGF is more likely to be used by the local or primary/secondary hospitals, and medical aid patients are more likely to visit local clinics rather than tertiary or teaching hospitals, our results can be interpreted based on the high correlation of the utilisation pattern of the medical aid patients and the types of drugs used by the medical institution ${ }^{33}$

Although not significantly different, the tonometry utilisation pattern in the DEX-implant group was higher than that in the anti-VEGF group. This finding might be related to the ocular safety profile of the DEX-implant. 'Intraocular pressure increase' is an expected complication of corticosteroid treatment, such as the
DEX-implant. ${ }^{16}$ The difference in the tonometry utilisation pattern was predictable. Nonetheless, our analysis should be interpreted with caution, and further longterm study is needed to investigate the incidence of cataract and cataract-related healthcare utilisation in two treatment groups.

Additionally, since our study period was rather short (30 months), we had a short follow-up period and a limited preindex period, which prevented us from selecting newly diagnosed patients with DME for our analyses. Thus, the disease duration and clinical characteristics of our patients were diverse. Although no significant difference was found in terms of the CCI score in our analysis, further long-term study is needed to compare the two cohorts.

Healthcare cost data are usually skewed, and various measures, such as transformation or non-parametric methods, were considered. However, non-parametric methods also have their own drawbacks, ${ }^{25}$ and using a

Table 3 Medical Institute visit days for patients with DME who received either anti-VEGF agents or a DEX-implant during the follow-up period

\begin{tabular}{|c|c|c|c|c|c|c|c|c|}
\hline & \multicolumn{2}{|c|}{ Mean (days) } & \multirow[t]{2}{*}{ P value* $^{*}$} & \multicolumn{2}{|c|}{ Median (days) } & \multirow[t]{2}{*}{$\mathrm{P}$ valuet } & \multirow{2}{*}{$\begin{array}{l}\text { Unadjusted } \\
\text { Visit ratio§ }(95 \% \mathrm{Cl})\end{array}$} & \multirow{2}{*}{$\begin{array}{l}\text { Adjusted } \neq \\
\text { Visit ratio }(95 \% \mathrm{Cl})\end{array}$} \\
\hline & Anti-VEGF & $\begin{array}{l}\text { DEX- } \\
\text { implant }\end{array}$ & & $\begin{array}{l}\text { Anti- } \\
\text { VEGF }\end{array}$ & $\begin{array}{l}\text { DEX- } \\
\text { implant }\end{array}$ & & & \\
\hline Total visits & 55.59 & 51.45 & 0.3124 & 39.00 & 40.00 & 0.5176 & 1.08 (1.06 to 1.11$)$ ๆ & 1.07 (1.04 to 1.09$)$ १ \\
\hline Outpatient visits & 45.26 & 44.20 & 0.7074 & 36.50 & 37.00 & 0.5787 & 1.02 (1.00 to 1.05$)$ & 1.05 (1.02 to 1.07$)$ १ \\
\hline Eye care-related visits & 15.17 & 15.85 & 0.3449 & 14.00 & 14.00 & 0.4977 & 0.96 (0.92 to 1.00$)$ & $0.96(0.92$ to 1.00$)$ \\
\hline $\begin{array}{l}\text { Outpatient eye care- } \\
\text { related visits }\end{array}$ & 14.97 & 15.14 & 0.8028 & 14.00 & 13.00 & 0.9359 & 0.99 (0.95 to 1.03$)$ & 0.99 (0.95 to 1.04$)$ \\
\hline
\end{tabular}

Anti-VEGF includes ranibizumab and aflibercept. The total visits to medical care are the aggregated number of visits to all medical care used by the patient during a 1 year postindex period.

*Estimated $p$ values based on the t-test.

†Estimated $p$ values based on the Wilcoxon test.

$\ddagger$ Adjusted for baseline characteristics such as age, gender, $\mathrm{CCl}$ and health plan type.

$\S$ The reference values obtained from the DEX-implant as 1.

$\mathbb{9 P}<0.001$

anti-VEGF, antivascular endothelial growth factor; DEX-implant, dexamethasone intravitreal implant; DME, diabetic macular oedema. 
Table 4 Comparison of healthcare resource use related to ophthalmological diagnosis during the follow-up for patients with DME who received either anti-VEGF agents or a DEX-implant

\begin{tabular}{|c|c|c|c|c|}
\hline & \multicolumn{2}{|l|}{ Mean ( $(\mathrm{SD})$} & \multicolumn{2}{|l|}{$P$ value } \\
\hline & Anti-VEGF & DEX-implant & Parametric* & Non-parametric† \\
\hline Optical coherence tomography & $7.09( \pm 3.38)$ & $7.43( \pm 3.15)$ & 0.2383 & 0.3623 \\
\hline Fluorescein angiography & $1.23( \pm 0.56)$ & $1.23( \pm 0.48)$ & 0.9852 & 0.6477 \\
\hline Fundus examination & $9.25( \pm 5.02)$ & $9.73( \pm 4.55)$ & 0.2535 & 0.1126 \\
\hline Fundus photography & $6.2( \pm 3.80)$ & $6.47( \pm 3.86)$ & 0.4571 & 0.4459 \\
\hline Tonometry & $12.08( \pm 6.09)$ & $13.21( \pm 7.09)$ & 0.0502 & 0.1191 \\
\hline Slit-lamp biomicroscopy & $12.4( \pm 6.01)$ & $13.15( \pm 6.61)$ & 0.1893 & 0.2375 \\
\hline Intravitreal injection & $2.69( \pm 2.29)$ & $2.089( \pm 1.37)$ & 0.001 & 0.0044 \\
\hline
\end{tabular}

Anti-VEGF includes ranibizumab and aflibercept.

${ }^{*}$ Estimated based on the t-test.

†Estimated based on the Wilcoxon test.

anti-VEGF, antivascular endothelial growth factor; DEX-implant, dexamethasone intravitreal implant; DME, diabetic macular oedema.

parametric approach to estimate the cost is endorsed. ${ }^{27}$ Thus, both parametric and non-parametric approaches were used in our analysis. To compare the healthcare costs or visiting days between the two groups, we applied a parametric approach such as an independent two-sample t-test. However, because these variables are usually skewed, we also used non-parametric methods and gamma GLMs with a $\log$ link in our analysis.

Previous studies examined various clinically relevant outcomes, such as visual acuity or central subfield thickness, ${ }^{13}{ }^{30}$ but clinical information is not available in the administrative claim database. Thus, we cannot conclude whether the reduced medical utilisation in the DEX-implant group could compromise the clinical outcome (such as aggravated visual acuity). According to Iglicki and colleagues, ${ }^{34}{ }^{35}$ DEX-implant treatment is effective for improving vision in patients with DME and delaying progression to proliferative diabetic retinopathy during long-term follow-up (24 months), suggesting that the DEX-implant might be clinically beneficial as well as economically attractive.

Moreover, our study is the first population-based study in Asia, which describes the characteristics of patients with DME and their healthcare resource utilisation patterns.

Our study showed the relative frequency of intravitreal injection of two treatment groups using population-based, real-world data. The frequency of the injection can be associated with patients' treatment outcomes as well as the patients' attitude (convenience) towards the treatment. ${ }^{36}$ Although the treatment regimen should be determined based on the clinical characteristics of each individual patient, providing the trend of the injection in two treatment groups could give clinicians a reference when they make decisions among many alternatives. Also, our findings could be attractive to policy makers who set priorities under budget constraint.

\section{CONCLUSION}

There was no significant difference in the demographic characteristics between the anti-VEGF and DEX-implant groups, and the DME-related healthcare resource utilisation of anti-VEGF users was higher than that of DEX-implant users. Specifically, the number of intravitreal injections and eye care-related medical costs for the DEX-implant group were lower than those of the antiVEGF group. Further long-term studies are needed to investigate the impact of cataract, which is a side effect of the DEX-implant, and to enhance our findings on the relative utilisation of the two groups.

\section{Author affiliations}

${ }^{1}$ College of Pharmacy, Ewha Womans University, Seoul, The Republic of Korea ${ }^{2}$ Department of Ophthalmology, Soonchunhyang University Hospital Seoul, Yongsangu, The Republic of Korea

${ }^{3}$ Department of Ophthalmology, Asan Medical Center, Songpa-gu, The Republic of Korea

${ }^{4}$ Department of Statistics, Ewha Womans University, Seoul, The Republic of Korea ${ }^{5}$ Department of Health Convergence, Ewha Womans University, Seoul, The Republic of Korea

${ }^{6}$ Allergan Korea Ltd, Seocho-gu, The Republic of Korea

Contributors $\mathrm{HC}$ conducted the statistical analysis and interpretation and drafted the article. HC and SB conceived the study and designed the analysis. KSC, JYL and $\mathrm{YL}$ defined the DME cohort and provided clinical advice on the design of the study. N-KC and DL supervised the statistical analysis of the data. All of the authors approved the final version of the article.

Funding This study was financially supported by Allergan Korea.

Competing interests The funding body has not been involved with the study design or the conduct of the study. YL was employed by Allergan Korea, but her input was based on her personal opinions.

Patient consent for publication Not required.

Provenance and peer review Not commissioned; externally peer reviewed.

Data availability statement Data may be obtained from a third party and are not publicly available.

Open access This is an open access article distributed in accordance with the Creative Commons Attribution Non Commercial (CC BY-NC 4.0) license, which permits others to distribute, remix, adapt, build upon this work non-commercially, and license their derivative works on different terms, provided the original work is 
properly cited, appropriate credit is given, any changes made indicated, and the use is non-commercial. See: http://creativecommons.org/licenses/by-nc/4.0/.

\section{REFERENCES}

1. Varma R, Bressler NM, Doan QV, et al. Prevalence of and risk factors for diabetic macular edema in the United States. JAMA Ophthalmol 2014;132:1334-40.

2. Klein R, Klein BE, Moss SE, et al. The Wisconsin epidemiologic study of diabetic retinopathy. XV. The long-term incidence of macular edema. Ophthalmology 1995;102:7-16.

3. Klein R, Knudtson MD, Lee KE, et al. The Wisconsin epidemiologic study of diabetic retinopathy XXIII: the twenty-five-year incidence of macular edema in persons with type 1 diabetes. Ophthalmology 2009;116:497-503.

4. International Diabetes Federation. IDF diabetes atlas. Brussels: international diabetes Federation, 2017. Available: http://www. diabetesatlas.org [Accessed 1 Aug 2018].

5. Korean Diabetes Association. Diabetes fact sheet in Korea, 2018. Available: http://www.diabetes.or.kr/bbs/index.html?sub_menu=\& code $=$ e_resource\&category $=1 \&$ gubun $=\&$ page $=1 \&$ number $=381 \&$ mode $=$ view\&order $=\&$ sort $=\&$ keyfield $=\& k e y=[$ Accessed 27 Jul 2019].

6. Gonder JR, Walker VM, Barbeau M, et al. Costs and quality of life in diabetic macular edema: Canadian burden of diabetic macular edema observational study (C-REALITY). J Ophthalmol 2014;2014:1-9.

7. Huang ES, Brown SES, Ewigman BG, et al. Patient perceptions of quality of life with diabetes-related complications and treatments. Diabetes Care 2007;30:2478-83.

8. Hariprasad SM, Mieler WF, Grassi M, et al. Vision-related quality of life in patients with diabetic macular oedema. Br J Ophthalmol 2008;92:89-92.

9. Kiss S, Chandwani HS, Cole AL, et al. Comorbidity and health care visit burden in working-age commercially insured patients with diabetic macular edema. Clin Ophthalmol 2016;10:2443-53.

10. Chen E, Looman M, Laouri M, et al. Burden of illness of diabetic macular edema: literature review. Curr Med Res Opin 2010;26:1587-97.

11. Shea AM, Curtis LH, Hammill BG, et al. Resource use and costs associated with diabetic macular edema in elderly persons. Arch Ophthalmol 2008;126:1748-54.

12. Mitchell P, Wong TY, Diabetic Macular Edema Treatment Guideline Working Group. Management paradigms for diabetic macular edema. Am J Ophthalmol 2014;157:505-13.

13. Gillies MC, Lim LL, Campain A, et al. A randomized clinical trial of intravitreal bevacizumab versus intravitreal dexamethasone for diabetic macular edema: the BEVORDEX study. Ophthalmology 2014;121:2473-81.

14. Schmidt-Erfurth U, Garcia-Arumi J, Bandello F, et al. Guidelines for the management of diabetic macular edema by the European Society of retina specialists (EURETINA). Ophthalmologica 2017;237:185-222.

15. Nguyen QD, Brown DM, Marcus DM, et al. Ranibizumab for diabetic macular edema: results from 2 phase III randomized trials: rise and ride. Ophthalmology 2012;119:789-801.

16. Boyer DS, Yoon YH, Belfort R, et al. Three-Year, randomized, shamcontrolled trial of dexamethasone intravitreal implant in patients with diabetic macular edema. Ophthalmology 2014;121:1904-14.

17. Antonetti DA, Barber AJ, Hollinger LA, et al. Vascular endothelial growth factor induces rapid phosphorylation of tight junction proteins occludin and zonula occluden 1. A potential mechanism for vascular permeability in diabetic retinopathy and tumors. J Biol Chem 1999;274:23463-7.

18. Heath Insurance Review \& Assessment Service. National drug formulary list. Available: http://www.hira.or.kr/bbsDummy.do?pgmid= HIRAA030014050000 [Accessed 9 Aug 2019].

19. Fraser-Bell S, Lim LL, Campain A, et al. Bevacizumab or dexamethasone implants for DME: 2-year results (the BEVORDEX study). Ophthalmology 2016;123:1399-401.

20. Korobelnik J-F, Do DV, Schmidt-Erfurth U, et al. Intravitreal aflibercept for diabetic macular edema. Ophthalmology 2014;121:2247-54.

21. Brown DM, Nguyen QD, Marcus DM, et al. Long-Term outcomes of ranibizumab therapy for diabetic macular edema: the 36-month results from two phase III trials: rise and ride. Ophthalmology 2013;120:2013-22.

22. Aljunid SM, Srithamrongsawat S, Chen W, et al. Health-Care data collecting, sharing, and using in Thailand, China mainland, South Korea, Taiwan, Japan, and Malaysia. Value Health 2012;15(1 Suppl):S132-S138.

23. Kim JA, Yoon S, Kim LY, et al. Towards Actualizing the value potential of Korea health insurance review and assessment (HIRA) data as a resource for health research: strengths, limitations, applications, and strategies for optimal use of HIRA data. J Korean Med Sci 2017;32:718-28.

24. Charlson ME, Pompei P, Ales KL, et al. A new method of classifying prognostic comorbidity in longitudinal studies: development and validation. J Chronic Dis 1987;40:373-83.

25. Briggs A, Gray A. The distribution of health care costs and their statistical analysis for economic evaluation. J Health Serv Res Policy 1998;3:233-45.

26. Qualls LG, Hammill BG, Wang F, et al. Costs of newly diagnosed neovascular age-related macular degeneration among Medicare beneficiaries, 2004-2008. Retina 2013;33:854-61.

27. Thompson SG, Barber JA. How should cost data in pragmatic randomised trials be analysed? BMJ 2000;320:1197-200.

28. Song S, Kim D. Analysis of healthcare utilization pattern of dibaetes patients: 10-year analysis National Health Insurance Ilsan Hospital; 2015.

29. Park KH, Kim YY, Jo YJ, et al. Healthcare utilization and treatment patterns in diabetic macular edema in Korea: a retrospective chart review. J Korean Med Sci 2019;34:e118.

30. Shah SU, Harless A, Bleau L, et al. Prospective randomized subjectmasked study of intravitreal bevacizumab monotherapy versus dexamethasone implant monotherapy in the treatment of persistent diabetic macular edema. Retina 2016;36:1986-96.

31. Tomkins-Netzer O, Taylor SRJ, Bar A, et al. Treatment with repeat dexamethasone implants results in long-term disease control in eyes with noninfectious uveitis. Ophthalmology 2014;121:1649-54.

32. Wells JA, Glassman AR, Ayala AR, et al. Aflibercept, bevacizumab, or ranibizumab for diabetic macular edema. $N$ Engl J Med 2015;372:1193.

33. Macular edema market understanding study by Hankook research November 16th; 2018.

34. Iglicki M, Busch C, Zur D, et al. Dexamethasone implant for diabetic macular edema in naive compared with refractory eyes: the International retina group real-life $24-$ month multicenter study. The IRGREL-DEX study. Retina 2019;39:44-51.

35. Iglicki M, Zur D, Busch $\mathrm{C}$, et al. Progression of diabetic retinopathy severity after treatment with dexamethasone implant: a 24-month cohort study the 'DR-Pro-DEX Study'. Acta Diabetol 2018;55:541-7.

36. Osterberg L, Blaschke T. Adherence to medication. N Engl J Med Overseas Ed 2005;353:487-97. 